\title{
Incorporating multiple-marker information to detect risk loci for rheumatoid arthritis
}

\author{
Xuexia Wang ${ }^{1,2}$, Huaizhen Qin ${ }^{1}$ and Qiuying Sha*
}

\author{
Addresses: ${ }^{1}$ Department of Mathematical Sciences, Michigan Technological University, Houghton, Michigan 49931, USA and \\ ${ }^{2}$ Capital University of Economics and Business, Beijing 100026, PR China \\ E-mail: Xuexia Wang - wxuexia@mtu.edu; Huaizhen Qin - hqin@mtu.edu; Qiuying Sha* - qsha@mtu.edu \\ ${ }^{*}$ Corresponding author
}

from Genetic Analysis Workshop 16

St Louis, MO, USA 17-20 September 2009

Published: 15 December 2009

BMC Proceedings 2009, 3(Suppl 7):S28 doi: 10.1186/1753-656I-3-S7-S28

This article is available from: http://www.biomedcentral.com/I753-656I/3/S7/S28

(C) 2009 Wang et al; licensee BioMed Central Ltd.

This is an open access article distributed under the terms of the Creative Commons Attribution License (http://creativecommons.org/licenses/by/2.0), which permits unrestricted use, distribution, and reproduction in any medium, provided the original work is properly cited.

\begin{abstract}
In genome-wide association studies, new schemes are needed to incorporate multiple-locus information. In this article, we proposed a two-stage sliding-window approach to detect associations between a disease and multiple genetic polymorphisms. In the proposed approach, we measured the genetic association between a disease and a single-nucleotide polymorphism window by the newly developed likelihood ratio test-principal components statistic, and performed a sliding-window technique to detect disease susceptibility windows. We split the whole sample into two sub-samples, each of which contained a portion of cases and controls. In the first stage, we selected the top $R$ windows by the statistics based on the first sub-sample, and in the second stage, we claimed significant windows by false-discovery rate correction on the $p$-values of the statistics based on the second sub-sample. By applying the new approach to the Genetic Analysis Workshop 16 Problem I data set, we detected 212 out of 531,601 windows to be responsible for rheumatoid arthritis. Except for chromosomes 4 and 18, each of the other 20 autosomes was found to harbor risk windows. Our results supported the findings of some rheumatoid arthritis susceptibility genes identified in the literature. In addition, we identified several new single-nucleotide polymorphism windows for follow-up studies.
\end{abstract}

\section{Background}

Rheumatoid arthritis (RA) is a common chronic destructive disease of an unknown complex etiology. Both genetic and environmental bases are thought to contribute to this disease. The human leukocyte antigen (HLA) region major histocompatibility complex (MHC) on chromosome $6(6 \mathrm{p} 21.3)$ is known to be associated with RA. This region is the only one that has been consistently shown to be both linked and associated with RA across all populations. It extends over $3.6 \mathrm{Mb}$ and is divided into three sub-regions (classes I, II, and III). It is a highly dense area containing about 220 genes, many of which are thought to have immunoregulatory functions [1]. Recently, matured genotyping technology and availability of large case-control collections have made it possible to detect mild risk loci. The Genetic 
Analysis Workshop 16 (GAW16) Problem 1 data set is such a large scale case-control study which contains genotypes at 531,689 single-nucleotide polymorphisms (SNPs) on chromosomes 1-22 for 868 cases and 1194 controls.

Recently, many approaches, e.g., Hotelling's $T^{2}$ test $[2,3]$ and the linkage disequilibrium (LD) contrast tests $[4,5]$, have been proposed to detect multiple-marker association. The Hotelling's $T^{2}$ test and the LD contrast test compare the means and the variance-covariance matrices of genotype scores between cases and controls, respectively. More recently, we proposed a likelihood ratio testprincipal component (LRT_PC) to compare the means and the variance-covariance matrices of genotype scores simultaneously [6]. However, all of these approaches only allow a SNP region of several to tens of markers.

In this article, we report a novel genome-wide slidingwindow approach to detect genetic association between a trait and SNP regions. This approach integrated the LRT_PC with the concept of sliding window [7] and the basic idea of two-stage approaches [8]. Applied to the GAW16 Problem 1 data set, our approach yielded results that support the findings in the literature of some RA susceptibility genes on chromosomes 1, 2, and 6 and detected more SNP windows for follow-up studies.

\section{Methods \\ LRT_PC statistic}

We recently proposed a LRT_PC approach to test the association between a given SNP window and a disease status [6]. To calculate the test statistic, we first perform principal component (PC) analysis to the genotype scores of the sampled individuals. Then, the LRT_PC test statistic is given by $L R T_{-} P C=(m+n) \log \left|\hat{\Sigma}_{p o o l_{-} p c}\right|-n \log \left|\hat{\Sigma}_{\text {case }_{-} p c}\right|-m \log \left|\hat{\Sigma}_{\text {control }} p c\right|$, where $n$ and $m$ are the numbers of cases and controls, respectively, and the $\hat{\Sigma}$ values are the sample variancecovariance matrices of the first $K$ PCs in cases, controls, and the pooled sample, respectively. Wang et al. [6] showed that the LRT_PC test is more powerful than the Hotelling's $T^{2}$ test and the LD contrast test [2-5] in most cases. The power of the LRT_PC test is perhaps due to its ability to capture the differences of the means and the variance-covariance matrices of genotype scores in cases and controls simultaneously.

\section{Two-stage sliding-window approach}

Because the LRT_PC test may be more powerful than other multi-marker tests, we wanted to use it to analyze the data set of GAW16 Problem 1. However, the LRT_PC can only be applied to a small chromosome region. To apply the LRT_PC to genome-wide association studies, we propose a sliding-window approach [7]. To use sliding windows, we divide all SNPs into contiguous overlapping windows and apply the LRT_PC in each window. Suppose that we use windows with a window size of $S$, then, all the SNPs can be divided into windows 1 to $S, 2$ to $S+1,3$ to $S+2$, and so on.

Because we do not know the distribution or asymptotic distribution of the test statistic LRT_PC, we need to use a permutation approach to estimate the $p$-value of the test. For a genome-wide association study, the number of windows usually is more than 500,000 and the number of permutations usually is no less than $1000(100,000$ permutations were used in this study). The computation is not feasible for the sliding-window approach discussed above. Thus, we propose a two-stage approach. In the two-stage approach, we split all individuals into two sub-samples. In the first stage, by assuming that all individuals are genotyped at all SNPs, we use the first sub-sample to select $R$ most promising SNP windows with the largest values of the LRT_PC statistic calculated via the first sub-sample. In the second stage, only the genotypes at SNPs within the $R$ most promising windows are used. In this stage, we use the second sub-sample to assess $P$ values for the $R$ selected windows by permutations and claim significance by the falsediscovery rate (FDR) correction in Benjamini and Hochberg [9]. For the two-stage approach, we only need to do permutations in the second stage. Thus, the two-stage approach is computationally much more efficient than one-stage approach.

To analyze the data set of GAW16 Problem 1 using LRT_PC based two-stage sliding-window approach, we use the following settings: window size is 5; the number of windows selected in the first-stage, $R$, is 1000; the sample size of the first sub-sample is $15 \%$ of the total sample (15\% cases and 15\% controls). In the first stage, the number of PCs used in the LRT_PC test in each window is 5, i.e., we do not perform PC analysis. In the second-stage, the number of PCs used in the LRT_PC test in each window, $K$, is decided by the fact that the first $K$ PCs can explain $85 \%$ of the total variability.

To choose the sample size of the first sub-sample, we did a power analysis based on a single-marker test similar to that of Wang et al. [8]. Our results showed that the optimal value of the sample size of the first sub-sample is between $10 \%$ and $30 \%$ of the total sample. We use the results based on a single-marker test as a reference to choose the sample size of the first sub-sample in this study (15\% of the total sample).

As pointed by Skol et al. [10], our proposed two-stage approach may be not as powerful as joint analysis. 
However, the results of Skol et al. also showed that when the sample size of the first sub-sample is small (15\% of the total sample), the power difference between the twostage approach and joint analysis is also small. To compare the power of the two-stage and one-stage approaches, we have done a small scale simulation study $(10,000$ SNPs and 1000 permutations). The simulation results showed that when the first subsample is $15 \%$ of the total sample, the power difference between the two approaches is also small. In summary, compared with the joint analysis and one-stage approach, our proposed two-stage approach has a small power loss in exchange for a big increase in computational efficiency.

\section{Results}

We applied the proposed two-stage sliding-window approach to analyze the RA data set from GAW16 Problem 1. After removing duplicated and contaminated samples, this data set contained genotypes at 531,689 SNPs on chromosomes 1-22 for 868 cases and 1194 controls. The missing genotypes were imputed using the method of Browning and Browning [11]. First, we used the first sub-sample to calculate the LRT_PC statistic for each of the 531,601 windows and selected 1000 of the most promising windows with the largest values of the LRT_PC test statistic. Then, we used the second subsample to calculate the LRT_PC test statistic for each of the 1,000 selected windows and used 100,000 permutations to evaluate the $p$-values. In total, we discovered 212 significant windows by the FDR correction at a nominal level 0.05, among which 126 windows are on chromosome 6. Many of the 212 windows are overlapped, especially for the windows on chromosome 6. After merging the overlapped windows, there were 68 nonoverlapped windows left. Among the 68 windows, 26 of them were on chromosome 6 . The 26 windows on chromosome 6 were in a region $(28,292,350$ to $33,349,147 \mathrm{bp}$ ) in high LD with HLA-DRB1, a factor known to have a strong association with RA. The details of these non-overlapped windows (except those on chromosome 6) are summarized in Table 1.

For validation purposes, we used the SNP Search Engine to find genes which contain or are near to SNPs that were discovered. We found rs2357135 nesting at "2q32", which is near to gene "STAT 4" and thus supported the finding in Remmers et al. [12]. In addition, our discoveries supported the findings of FCRL3 on chromosome 1 [13] and HLA region (MHC) on chromosome 6 [1]. Additionally, we detected many novel risk windows for follow-up studies. Except for chromosomes 4 and 18, each of the other autosomes was found to carry risk windows. For example, we detected SNP rs2047465 which nests in gene SDK2 on chromosome 17.
Table I: The details of non-overlapped significant windows

\begin{tabular}{|c|c|c|c|c|}
\hline $\begin{array}{l}\text { Window } \\
\text { ID }\end{array}$ & Chr & Physical Location & Genes & References \\
\hline 1 & 1 & $792429,107 \mid 463$ & $\begin{array}{l}\text { FAM87B, } \\
\text { Clorf159 }\end{array}$ & \\
\hline 2 & I & |49769454, I49786537 & FCRL3 & [13] \\
\hline 3 & 2 & 139347733, 139370993 & $\mathrm{NXPH} 2$ & \\
\hline 4 & 2 & $|58| 03376,|58| 24755$ & CYTIP & \\
\hline 5 & 2 & 192062916,192117323 & MYOIB & \\
\hline 6 & 2 & |93087360, |931|709| & STAT4 & {$[12]$} \\
\hline 7 & 2 & 204487030,204527849 & & \\
\hline 8 & 2 & 217410540,217413523 & & \\
\hline 9 & 3 & 61980695,61998337 & PTPRG & \\
\hline 10 & 3 & II 2000554, II 2031324 & & \\
\hline II & 5 & 25934777, 25967I9I & & \\
\hline 12 & 5 & 111055309,111062116 & & \\
\hline 13 & 5 & $|376| 4229, \mid 37669929$ & GFRA3 & \\
\hline 14 & 5 & $175278829, \mid 75508504$ & & \\
\hline 15 & 7 & 35309745,35321578 & & \\
\hline 16 & 8 & 20380853,20411350 & & \\
\hline 17 & 9 & 34654488,34675940 & CCL27 & \\
\hline 18 & 9 & $84 I 7940 I, 84220779$ & SLC28A3 & \\
\hline 19 & 9 & 91845119,91920813 & & \\
\hline 20 & 10 & 5016096,5053944 & & \\
\hline 21 & 10 & 49685217,49698112 & WDFY4 & \\
\hline 22 & 10 & 87994785,88004329 & GRIDI & \\
\hline 23 & 11 & $67868248,67886 \mid 82$ & LRP5 & \\
\hline 24 & 11 & 68807443, 6882532I & & \\
\hline 25 & 12 & 130500249,130524477 & & $\begin{array}{l}\text { hypothetical } \\
\text { LOCI I } 6437\end{array}$ \\
\hline 26 & 13 & $734 I I I 8 I, 73419864$ & $K L F / 2$ & \\
\hline 27 & 13 & 113652806,113781019 & FAM70B & \\
\hline 28 & 14 & 31921536,31942503 & AKAP6 & \\
\hline 29 & 14 & 80898925,80930993 & STON2 & \\
\hline 30 & 15 & $72678|84,7272| 493$ & & \\
\hline 31 & 16 & 2452524,2582219 & Cl6orf59 & \\
\hline 32 & 16 & 9214168,9232588 & & \\
\hline 33 & 16 & $1265 \mid 732,12676977$ & & \\
\hline 34 & 16 & 64715655,64730155 & & \\
\hline 34 & 16 & $647 \mid 5655,64730155$ & & \\
\hline 35 & 16 & 64735494,64764278 & & \\
\hline 36 & 16 & $6745|454,675| 4 \mid 26$ & TMCO7 & \\
\hline 37 & 17 & 34194598,34251205 & PIP4K2B & \\
\hline 38 & 17 & 68919134,68930918 & SDK2 & \\
\hline 39 & 19 & $4|254897,4| 282169$ & WDR62 & \\
\hline 40 & 22 & 24746347,24757608 & MYOI8B & \\
\hline 41 & 22 & 2476084I, 24782942 & & \\
\hline 42 & 22 & 43076969,43084289 & & \\
\hline
\end{tabular}

Note: There are 26 significant non-overlapped windows on chromosome 6 which are in a region $(28,292,350$ to $33,349,147$ bp) in high LD with HLA-DRBI, a factor known to have a strong association with RA. We did not list those 26 non-overlapped significant windows due to space limitations.

\section{Discussion}

In this article, we proposed a two-stage sliding-window approach and, in each window, our recently proposed LRT_PC test was applied to test the association between a window and a disease. Then, we applied the method to GAW16 Problem 1 to detect risk windows for RA. Different existing RA association studies discovered diverse susceptibility genes on all chromosomes except for MHC candidate 
genes on chromosome 6. Our analysis supported the findings of some RA susceptibility genes that have been identified to be associated with RA in the literature.

It is intractable to formulate the null distribution of the LRT_PC statistic and thus the permutation approach must be applied to evaluate the $p$-values of top $R$ promising windows ( $R=1000$ was used in this study). For a given $R$, the number of permutations must be large enough to obtain accurate $p$-values. Thus, the choice of $R$ partially depends on computational capacity. Further efforts are needed to determine the optimal value of $R$.

In this study, we used 5 PCs, i.e., we did not perform PC analysis in LRT_PC test for each window of size 5 in the first stage. We did not perform PC analysis (or use 5 PCs) in the first stage for two reasons. One is that we have to use the same number of PCs in different windows so that the values of the test statistic in different windows are comparable. The other is that in the first stage, we only use the test statistic to rank the SNP windows and the results are similar when using different numbers of PCs (results not shown). Another remaining question regarding the proposed method is how to choose the window size. Most often, researchers use windows of size 3. In the LRT_PC test, we use PC analysis to reduce the dimension, and thus we can use a larger window size (i.e., window size of 5 ).

\section{Conclusion}

In this article, we proposed a two-stage sliding-window approach to detect the association between SNP windows and a disease status. Application to GAW16 Problem 1 data set illustrated its practical advantages. Our results supported the findings of several genes which were identified to be responsible for RA in the literature. We also discovered several additional SNP windows for follow-up studies.

\section{List of abbreviations used}

FDR: False-discovery rate; GAW16: Genetic Workshop Analysis 16; HLA: Human leukocyte antigen; LD: Linkage disequilibrium; LRT-PC: Likelihood ratio test-principal component; MHC: Major histocompatibility complex; PC: Principal component; RA: Rheumatoid arthritis; SNP: Single-nucleotide polymorphism.

\section{Competing interests}

The authors declare that they have no competing interests.

\section{Authors' contributions}

$\mathrm{XW}$ performed the statistical analysis and wrote the first draft of the manuscript. HQ participated in the study design and helped to draft the manuscript. QS contributed to the design of the study and to the manuscript preparation. All authors read and approved the final manuscript.

\section{Acknowledgements}

The Genetic Analysis Workshops are supported by NIH grant ROI GM031575 from the National Institute of General Medical Sciences. This work was supported by NIH grants ROI GM069940 and the OverseasReturned Scholars Foundation of Department of Education of Heilongjiang Province (II52HZOI).

This article has been published as part of BMC Proceedings Volume 3 Supplement 7, 2009: Genetic Analysis Workshop 16. The full contents of the supplement are available online at http://www.biomedcentral.com/ $1753-656 \mid / 3$ ? issue $=$ S7.

\section{References}

I. Newton JL, Harney SM, Wordsworth BP and Brown MA: A review of the MHC genetics of rheumatoid arthritis. Genes Immun 2004, 5:151-157.

2. Xiong $M$, Zhao J and Berwinkle E: Generalized $T^{2}$ test for genome association studies. Am J Hum Genet 2002, 70: $1257-1268$

3. Fan R and Knapp M: Genome association studies of complex diseases by case-control designs. Am J Hum Genet 2003, 72:850-868.

4. Zaykin DV, Westfall PH, Young SS, Karnoub MA, Wagner MJ and Ehm MG: Testing association of statistically inferred haplotypes with discrete and continuous traits in samples of unrelated individuals. Hum Hered 2002, 53:79-91.

5. Wang T, Zhu X and Elston RC: Improving power in contrasting linkage-disequilibrium patterns between cases and controls. Am J Hum Genet 2007, 80:91I-920.

6. Wang $X$, Zhang $S$ and Sha $Q$ : A new association test to test multiple-marker association. Genet Epidemiol 2009, 33:164-17|.

7. Yang $\mathrm{H}$, Lin $\mathrm{C}$ and Fann $\mathrm{C}$ : A sliding-window weighted linkage disequilibrium test. Genet Epidemiol 2006, 30:53I-545.

8. Wang H, Thomas DC, Peer I and Stram DO: Optimal two-stage genotyping designs for genome-wide association scan. Genet Epidemiol 2006, 30:356-368.

9. Benjamini $Y$ and Hochberg $Y$ : Controlling the false discovery rate: a practical and powerful approach to multiple testing. J Roy Stat Soc Ser B 1995, 57:289-300.

10. Skol AD, Scott LJ, Abecasis GR and Boehnke M: Joint analysis is more efficient than replication-based analysis for two-stage genome-wide association studies. Nat Genet 2006, 38:209-213.

II. Browning SR and Browning BL: Rapid and accurate haplotype phasing and missing-data inference for whole-genome association studies by use of localized haplotype clustering. Am J Hum Genet 2007, 81: 1084-1097.

12. Remmers EF, Plenge RM, Lee AT, Graham RR, Hom G, Behrens TW, de Bakker PI, Le JM, Lee HS, Batliwalla F, Li W, Masters SL, Booty MG, Carulli JP, Padyukov L, Alfredsson L, Klareskog L, Chen WV, Amos Cl, Criswell LA, Seldin MF, Kastner DL and Gregersen PK: STAT4 and the risk of rheumatoid arthritis and systemic lupus erythematosus. N Engl J Med 2007, 357:977-986.

13. Kochi Y, Yamada R, Suzuki A, Harley JB, Shirasawa S, Sawada T, Bae SC, Tokuhiro S, Chang X, Sekine A, Takahashi A, Tsunoda T, Ohnishi Y, Kaufman KM, Kang CP, Kang C, Otsubo S, Yumura W, Mimori A, Koike T, Nakamura $Y$, Sasazuki T and Yamamoto $K$ : A functional variant in FCRL3, encoding Fc receptor-like 3, is associated with rheumatoid arthritis and several autoimmunities. Nat Genet 2005, 37:478-485. 